\title{
Adaptive and dynamic characteristics in hybrid agile management model for software development project success
}

\begin{abstract}
The management of traditional software development methodologies and agile software development methodologies over the decades have not worked to its expectation in increasing the success rates of software projects. The Agile Manifesto in 2001 had promised to better manage IT projects with its 4 values and 12 principles but project success still appears to be elusive. The study and application of critical success and failure factors have been done by researchers but these factors are quick remedy solutions and not long-term robust improvements in the management of development methodologies. More research is required into the management of traditional and agile methodologies characteristics instead of success and failure factors. The coexistence of these characteristics as hybrid methods has also provided evidence of an increase in productivity and further research is required to strengthen and close current gaps in the management of hybrid methodologies. An empirical method with a quantitative approach is adopted to collect data with questionnaires from software development focus groups involved in industry projects. The data collected is analyzed using the Statistical Package for the Social Sciences (SPSS) technique and the Partial Least Squares (PLS) tool. The findings provide a set of significant characteristics of adaptive and dynamic features, which are combined with organizational objectives to prove there is a strong relationship between project success with hybrid methods and hybrid combination patterns.
\end{abstract}

Keyword: Project success; Software development; Agile; Methodologies; Adaptive; Dynamic; Hybrid 